\title{
MAPPING SYSTEM AND PHOTOGRAMMETRIC PROCESSING METHOD FOR TETHERED BALLOON PLATFORM
}

\author{
J.M. Li ${ }^{1,2}$, C. R. $\mathrm{Li}^{1, *}$, G. Z. $\mathrm{Su}^{3}$, W. $\mathrm{Li}^{1}$, L. L. Ma ${ }^{1}$, Y. K. Liu ${ }^{1}$ \\ ${ }^{1}$ Key Laboratory of Quantitative Remote Sensing Information Technology, Academy of Opto-Electronics, Chinese Academy of \\ Sciences, China - (jmli, crli, liwei, 1lma, liuyk)@aoe.ac.cn \\ ${ }^{2}$ University of Chinese Academy of Sciences, China \\ ${ }^{3}$ Chinese Academy of Surveying \& Mapping,China, - 174083588@qq.com
}

KEY WORDS: Tethered Balloon, Wide-Angle Camera, Multi-Altitude Photography, Photogrammetry

\begin{abstract}
:
As a platform with the advantages of safety, wide altitude range and long flight time, tethered balloon has been used in archaeology, coastal, island mapping and other related fields. Tethered balloon is tied to the mooring system by tether rope, and often moves in a small range with the airflow, resulting in a very close distance between the camera stations. Due to the small base-to-height ratio, failure of relative orientation or poor quality of point cloud tend to appear frequently. In this paper, a mapping system for tethered balloon is introduced which consists of a wide-angle camera, two auxiliary orientation cameras, and a dual GNSS/IMU system. The wide-angle camera combined by five cameras provides a larger observation range. Two auxiliary orientation cameras installed at the end of extension rods are designed to increase the baseline. The system should be operated during the taking-off, hovering and landing stages of the tethered balloon, so as to take images of different altitudes. The system has been adopted in the first experimental flight in 2018 to perform island topographic survey. Data processing method was applied to test the effect of the system. The method that choosing wide-angle images of different heights, and auxiliary images at low-altitude can be used for aerial triangulation successfully, and obtain point cloud with good quality. The results showed that by adding auxiliary cameras and taking images of different heights for data processing, the problem of small intersection angle could be overcome to some extent.
\end{abstract}

\section{INTRODUCTION}

Aerostat platform is a new type of aerial remote sensing and monitoring platform that relies mainly on air buoyancy to overcome its own gravity. It has the characteristics of safety, long flight time, and cost-effectiveness, which can be a supplement to other flight platforms. Aerostat platforms can be separated into two categories: (1) tethered balloons constrained by cables, (2) powered airships (Peng, 2019). Powered airship platforms are equipped with power propulsion devices, which fearures include cheap, easy to operate, can fly in low altitude and low speed. They have been widely used in mineral resources management, geological environment survey, disaster prevention, agriculture, forestry, water conservancy, etc. Example researches for the airships include the low-height photogrammetric studies by Zhang Y. J. and other researchers (Zhang, 2009; Peng, 2009), the photogrammetric documentation of archaeological sites by Bitelli G. (Bitelli, 2004), single linear hyperspectral pushbroom imaging sensor directly hung under the aerostat by Zhang A. W. (Zhang, 2015), and unmanned airship thermal infrared remote sensing system for monitoring of urban thermal environments by Ren P. (Ren, 2015).

The tethered balloons are tied to the ground by tether rope, that makes the observation range so limited. So the tethered balloons are usually used in archaeology and fixed-point monitoring. Cameras on tethered balloon move in a small range with the wind at a fixed altitude, resulting in a very close distance between the camera stations. The small base-to-height ratio will cause poor accuracy of elevation, and even failure of the relative orientation. In order to solve this problem, (MozasCalvache, 2012) used a manpower solution, in which three to

\footnotetext{
* Corresponding author: crli@aoe.ac.cn; Tel.: +86-010-8217-8634
}

five people pulled the tether rope to move the balloon to the designed camera station. The solution is not suitable for areas difficult to access, nor for large balloon platforms. (Altan, 2008) used GCPs(Ground Control Points) for the exterior orientation of images. To deal with some images which taken very closed to the object of interest, many control points had to be measured, which will be a big work and needed long work time .

In this paper, a mapping system is introduced for tethered balloon which consists of a wide-angle camera, two auxiliary orientation cameras, and a dual GNSS/IMU(Global Navigation Satellite System/Inertial Measurement Unit) system. The system works during the taking-off, hovering and landing stages of the tethered balloon, so as to take images of different altitudes. Then the photogrammetric processing method to solve the baseto-height ratio problem is also presented.

\section{MULTI-CAMERA MAPPING SYSTEM}

\subsection{System Design}

In this paper, a multi-camera mapping system is integrated into a tethered balloon, with a maximum payload capacity of $30 \mathrm{~kg}$. We needed the mapping system to create maps at a spatial resolution of at least $15 \mathrm{~cm}$ and with a georeferencing accuracy of $1 \mathrm{~m}$. There are several problems to be solved in the system design. They are the short baseline length of single camera, the narrow cover, and heading angle measurement under approximately static conditions.

The problem of base-to-height ratio makes it difficult to produce high-precision DSM (Digital Surface Model), thus limiting the accuracy of image rectification. In order to solve the problem, the mapping system which consisted by a wide- 
angle camera and auxiliary cameras is designed (Figure 1). The wide-angle camera is mounted in the main frame. The hanging plate adapter is installed on the main frame structure as an interface with the tethered balloon. Two triangular extension rods with a length of $1.8 \mathrm{~m}$ are installed on the wingspan directions of the main frame. The frame is made of magnesium- aluminium alloy to reduce the weight of the entire system. Two auxiliary orientation cameras are installed at the end of the extension rods. Dual GNSS/IMU system is used to acquire the position and attitude data. The two GNSS antennas are installed on the extension rods, while the IMU unit and the GNSS receiver unit are mounted on the main frame.

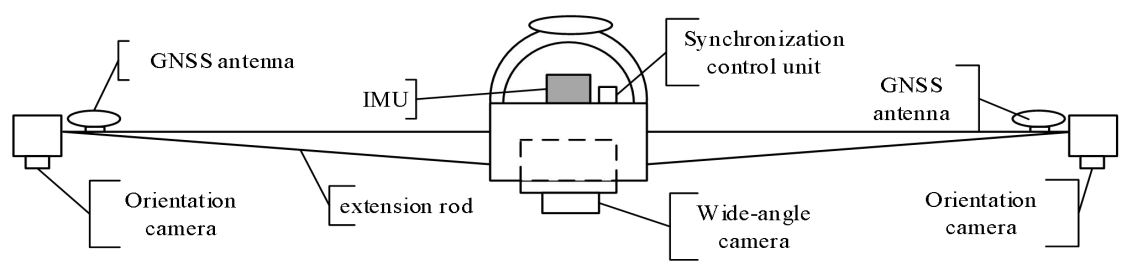

Figure 1. Schematic figure of the mapping system and its main components

\subsection{Imaging Module}

The imaging module (Table 1) is composed of a wide-angle camera and two auxiliary orientation cameras. The wide-angle camera is composed of five Canon cameras (Figure 2). The lens of each camera has a field of view (FOV) of $54.3^{\circ} \times 37.8^{\circ}$, and a focal length of $35 \mathrm{~mm}$. Given the focal length and the sensor size, the GSD is around $0.1 \mathrm{~m}$ for a flight altitude of $800 \mathrm{~m}$. The weight of each camera is $845 \mathrm{~g}$, and the whole weight of the wide-angle camera is about $13.5 \mathrm{~kg}$ (including the weight of the frame and the cable).

\begin{tabular}{|l|l|}
\hline \multicolumn{2}{|l|}{ wide-angle camera (Canon 5DS R) } \\
\hline $\begin{array}{l}\text { Number of cameras } \\
\text { focal length }\end{array}$ & 5 \\
Image size & $\begin{array}{l}(8688 \times 5792) \text { pixels } \\
\end{array}$ \\
& $(1 \times 0.68) \times$ flight altitude \\
\hline auxiliary camera (Canon $100 \mathrm{D})$ \\
\hline Number of cameras & 2 \\
focal length & $24 \mathrm{~mm}$ \\
Image size & $(5184 \times 3456)$ pixels \\
& $(1.05 \times 0.67) \times$ flight altitude \\
\hline
\end{tabular}

Table 1. Technical specifications of the imaging module

The wide-angle camera for tethered balloon has two advantages. First is that the field of view is wide, which means a larger observation range can be obtained. The second advantage is that wide field of view can improve the altitude accuracy effectively. In our experiment, the middle camera looks down vertically, and the other four cameras tilting in pairs. The advantage of this design is that the self-calibration of combined cameras can be realized by overlaps, and the installation deviation on three degrees of freedom can be detected. Therefore a soft style steady frame can be used to reduce the weight of the system. The FOV of the combined wide-angle image can reach $94^{\circ} \times 86^{\circ}$, and the image size is up to $17000 \times 15000$ pixels.
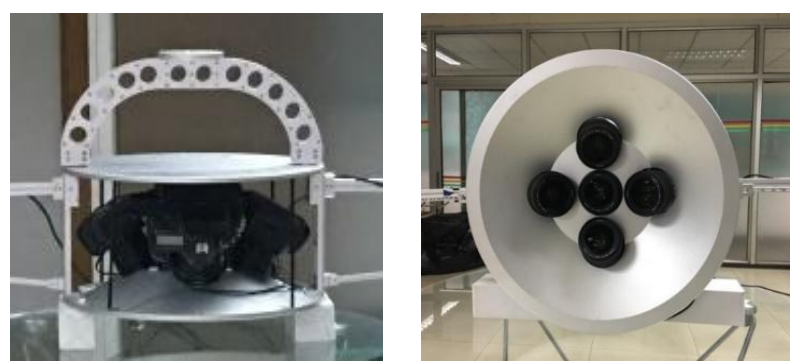

Figure 2. The wide-angle camera
Auxiliary orientation cameras are designed to extern the baseline length at a low flight height. Two extension rods with the length of $1.8 \mathrm{~m}$ are installed on both sides of the wide-angle camera. The auxiliary camera is placed at the end of each extension rod. Considering the stability of the mechanical structure, the small and lightweight camera is needed. Canon $100 \mathrm{D}$ is chosen as auxiliary orientation camera with a focal length of $24 \mathrm{~mm}$, and the FOV is $55.6^{\circ} \times 37.4^{\circ}$.

\subsection{Navigation Module}

The mapping system is equipped with a NovAtel IMU with 3axis gyroscopes, and 3-axis accelerometers. The position is recorded using a dual-frequency, dual-antenna RTK(Real Time Kinematics) GNSS receiver. GNSS/IMU data fusion uses a multisensor Kalman filter proposed by Inertial Explorer software. After post processing, the spatial accuracy can reach $0.02 \mathrm{~m}$, and the accuracy of roll \& pitch is about $0.007^{\circ}$.

The heading is measured by the dual GNSS antennas. Because of the motion pattern of tethered balloon, it's difficult to measure the attitude of heading using IMU. So dual GNSS antennas are necessary. The GNSS antennas are installed on the end of each extension rod along with the auxiliary orientation cameras. The baseline length of GNSS antennas is about $3.7 \mathrm{~m}$, which provides a heading estimation with accuracy of about $0.054^{\circ}$.

\subsection{Control Module}

Synchronization control unit is used to control the seven cameras to expose simultaneously. It receives the GNSS event, so that the synchronization is based on GPS timestamps.

The frame frequencies of all the cameras are set before the mission. The synchronization control unit read the configuration, and sent orders to the cameras. Then the pulses are sent by the cameras to the synchronization control unit upon the start of image exposure.

In order to cope with camera mechanical shutter delay, the synchronization control unit was designed to realize shutter delay compensation mechanism. By receiving the exposure feedback of each camera dynamically, the time delay of each camera is computed in real-time. The signal of exposure order is compensated dynamically during the whole flight.

The multi-cameras control and monitoring software coded in $\mathrm{C}++$ languages has been developed. It is installed in an Intel NUC (Next Unit of Computing), a small box-shaped computer. The control software of the Navigation Module is installed in the NUC too. The NUC is connected to the ground control part by a network line, allowing the operator on the ground to communicate with the imaging modules and the navigation 
modules, to check the status of the GNSS/IMU system and the cameras (Figure 3).

The images are recorded in camera memory cards, and the exposure time of each image is recorded by the synchronization control unit. The GNSS/IMU data is recorded by the NUC.

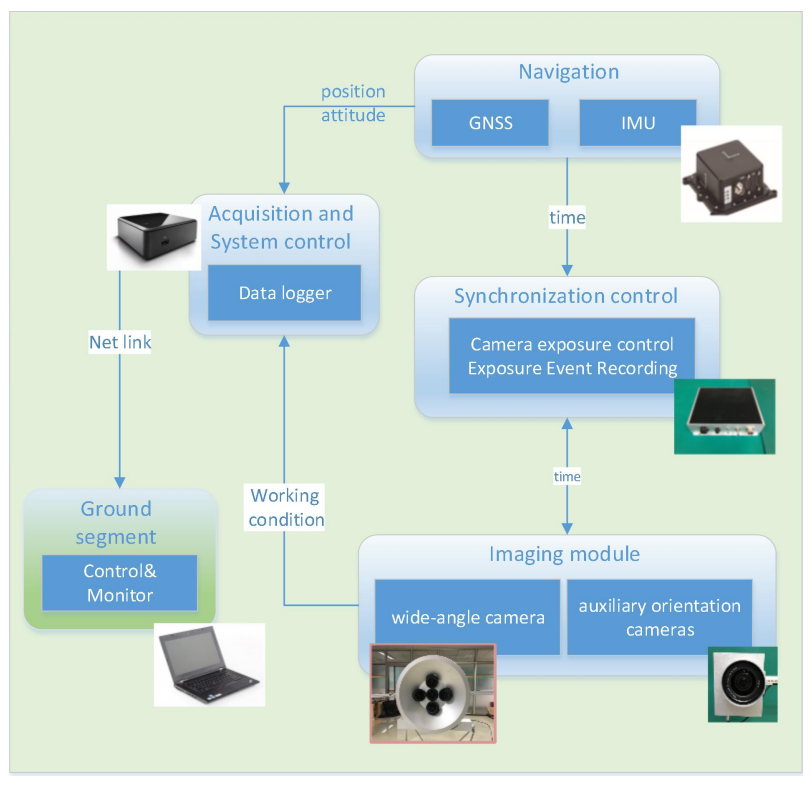

Figure 3. Diagram of the mapping system

\subsection{Ground Calibration}

Camera calibration is carried out on the ground to obtain the calibration parameters of each camera and the relative orientation elements of the five-combined cameras. Ground calibration site is performed on ground calibration site.

The focal length of each camera should be locked before ground camera calibration. Pictures of the calibration site are taken, making every corner of the image covered by the control points evenly. Based on the coordinates of the control points, the calibration parameters of each camera can be calculated.

The purpose of the relative calibration of the five-combined cameras is to find the relative orientation elements between the four oblique images and the center images. The small angle variation between the combined camera images has an important effect on the image points offset. By image matching, the parallax of the corresponding point in the overlap is calculated (Gui, 2009). Then the relative orientation angle elements are solved by least square method. Equation 1 is the parallax equation.

$$
\left\{\begin{array}{c}
\Delta x_{i j}=\frac{f^{2}+x_{\mathrm{i}}^{2}}{f} \Delta \varphi_{i}+\frac{x_{\mathrm{i}} \mathrm{y}_{\mathrm{i}}}{f} \Delta \omega_{i}+y_{i} \Delta \kappa_{i}-\frac{f^{2}+x_{j}{ }^{2}}{f} \Delta \varphi_{j}+\frac{x_{j} \mathrm{y}_{j}}{f} \Delta \omega_{j}+y_{j} \Delta \kappa_{j} \\
\Delta y_{i j}=\frac{x_{\mathrm{i}} \mathrm{y}_{\mathrm{i}}}{f} \Delta \varphi_{i}+\frac{f^{2}+x_{i}^{2}}{f} \Delta \omega_{i}+x_{i} \Delta \kappa_{i}-\frac{x_{j} \mathrm{y}_{j}}{f} \Delta \varphi_{j}-\frac{f^{2}+x_{j}{ }^{2}}{f} \Delta \omega_{j}-x_{j} \Delta \kappa_{j}
\end{array}\right.
$$

where $\quad i, j=$ camera numbers

$$
\begin{aligned}
& \left(x_{i}, y_{i}\right)=\text { image coordinates } \\
& \Delta x_{i j}, \Delta_{i j}=\text { image parallax } \\
& \Delta \varphi, \Delta \kappa, \Delta \omega=\text { orientation parameters }
\end{aligned}
$$

\section{FIELD OPERATION AND DATA PROCESSING}

\subsection{Field Operation}

As a payload of tethered balloon, the position of camera stations is determined by the airflow, which is not reliable. In order to increase the intersection angle, images at different flight heights are needed.

The flight of the mapping system has four stages. The mapping system is started in the balloon stationary stage, and the imaging modules and the navigation modules are configured and initialized. During the taking-off stage, images of different flight heights are collected. In the hover stage, the mapping system moves with the balloon platform within a small range. So the frame frequency of the camera should be reduced. Then images are taken during the landing stage similar to the taking off stage. Images at different altitudes are obtained until landing back to the ground.

\subsection{Photogrammetric processing}

In the data processing chain, the time synchronization relationship of the seven cameras and the GNSS/IMU system should be matched and established according to the exposure time firstly. Then the wide-angle images are generated by the groups of the five combined camera images. Using wide-angle images and auxiliary images acquired at different flight heights, image pairs with fixed baseline or different flight heights baseline are selected to construct stereo image models. At last, the point cloud and orthophoto images are produced.

Firstly, the GNSS RTK and GNSS/IMU data fusion using a multisensor extended Kalman filter is obtained by Inertial Explorer software. With the base station data, dual GNSS/IMU data measured during the flight, and the boresight parameters, the orientations of the GNSS/IMU unit are converted to Northing-Easting -Altitude-Omega-Phi-Kappa vectors with the frequency of $200 \mathrm{~Hz}$.

In traditional georeferencing processing, the event pulses are sent by the cameras to the navigation unit upon the start of image exposure, so the exterior orientation elements can be output associated with each aerial image directly by Inertial Explorer software. But our system contains seven cameras. It is difficult to send event to the navigation unit from each camera. The exposure time of seven cameras are recorded by the synchronization control unit, without communication with the navigation module. Therefore, time synchronization process for the images taken by seven cameras and the GNSS/IMU data is necessary. The timestamps are extracted from the synchronization control unit, and the trigger time intervals of images are calculated to find the first image of flight. Then, the exposure time recorded by Canon camera in image files is extracted, and the time intervals are calculated too. Compared with the time intervals calculated from the timestamps file and images, the images and the recorded timestamps with UTC time correspond one by one. Finally, according to the exposure time, the images of seven cameras are grouped, and the corresponding exterior orientation elements are interpolated based on the IMU/GNSS data.

The images taken by five-combined cameras are mosaicked to produce a wide-angle image. Four tilted images are corrected into horizontal images according to the relative orientation elements of five cameras. Due to the flutter of mechanical structure, exposure time difference and other reasons, there will still be residual errors when the five images of each group are mosaicked directly with calibration parameters. Therefore, relative relationship is needed to rebuild between each image group. The relative orientation is based on the initial value of the calibration parameters and the corresponding points in the overlaps. The tilted images are corrected to the horizontal images which use a same virtual coordinate system. Removing the uneven illumination phenomenon for the images, the images are mosaicked to a wide-angle image. 
Finally, appropriate images are selected for aerial triangulation processing. The criterion of image selection is enlarged base-toheight ratio. Wide angle images of different heights should be chosen to ensure a larger intersection angle, which improves the accuracy of aerial triangulation and forward intersection. The corresponding auxiliary images taken at low altitudes were selected, while those images taken at higher altitudes were removed which will reduce the accuracy of point cloud also because of the base-to-height ratio.

\section{EXPERIMENT AND DISCUSSION}

\subsection{Experimental Overview}

First test flight was performed above the coast of Sanya, China, in 2018. The mapping system was mounted on the tethered balloon as shown in Figure 4. The flight height was about $330 \mathrm{~m}$.

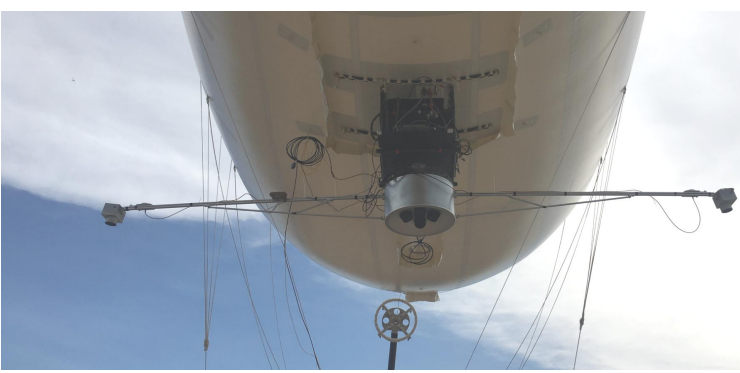

Figure 4. Mapping system mounted on the tethered balloon

The exposure frequency of each camera was set with the stage of the balloon platform. During the taking-off stage and the landing stage, the exposure interval was $3 \mathrm{~s}$, while at hover stage, the exposure interval was 10s. GCPs were measured before the flight by RTK GPS. Due to the limitation of topographic conditions, all the thirteen GCPs were located on one side of the flight area, while the other side was the sea.

\subsection{Results and Discussion}

In order to prove the validity of the system design, four different ways to select images were performed. Mode 1 was chosen at wide-angle images in hover phase, so the images were taken at the same height. in mode 2, wide-angle images from the takeoff, hover and landing phase were chosen to obtain images at different flight heights. Similarly mode 3 was chosen with wide-angle images and auxiliary images of different heights. Mode 4 chose wide-angle images of different heights, and auxiliary images at low-altitude (below $100 \mathrm{~m}$ ).

During the flight, the wind was relatively speed low, and the farthest distance between images taken during hover stage was about $10 \mathrm{~m}$. Because of the small base-height ratio, mode 1 was failed in aerial triangulation processing.

Both mode 2, 3, and 4 were succeeded in aerial triangulation processing, which proved that the method of increasing the intersection angle by height difference was effective. The RMS errors of the four modes are listed in Table 2.

\begin{tabular}{|c|c|c|c|}
\hline MODES & Error X (m) & Error Y (m) & Error Z (m) \\
\hline 1 & - & - & - \\
2 & 0.221 & 0.467 & 2.261 \\
3 & 0.400 & 0.857 & 3.315 \\
4 & 0.318 & 0.228 & 0.227 \\
\hline
\end{tabular}

Table 2. RMS errors of the four modes

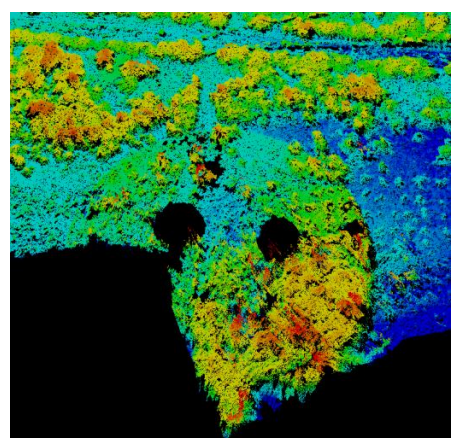

(a) mode 2

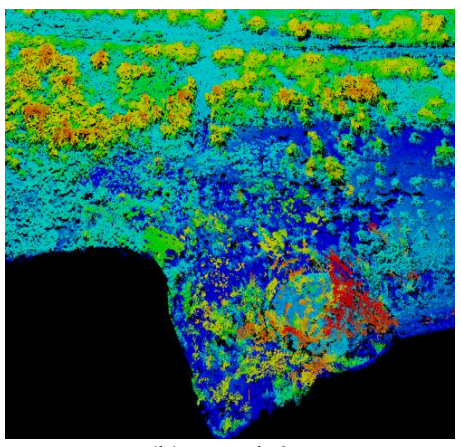

(b) mode3.

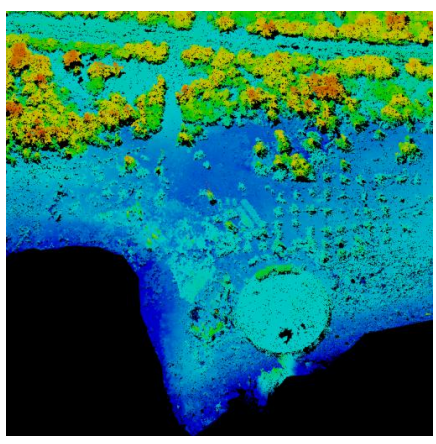

(c) mode4.

Figure 5.Point cloud produced by different modes(colored by elevation). (a)The point cloud is obviously wrong of mode 2. (b)There are lots of outliers in the point cloud of mode3. (c) The circular platform is clearly visible of mode 4.

Though the errors of $\mathrm{X}$ and $\mathrm{Y}$ direction are similar in the three modes, the errors of $\mathrm{Z}$ direction are different. Mode 4 is better than other modes. At the same time, the quality of point cloud was not good in mode 2 and mode 3 . There were lots of outliers in the point cloud, especially at the area just below the tethered balloon (Figure 5). In these areas, lacking of texture in beach and small intersection angle of the corresponding points leads to mismatching, which results in the poor quality of point cloud and the orthoimage.

Mode 4 produced the point cloud with better quality. Figure 6 shows the DSM and the rectified image. The positional error computed on GCPs was about $0.3 \mathrm{~m}$, and the elevation error was about $0.2 \mathrm{~m}$. The quality of aerial triangulation and forward intersection was improved by the low-altitude auxiliary images effectively.

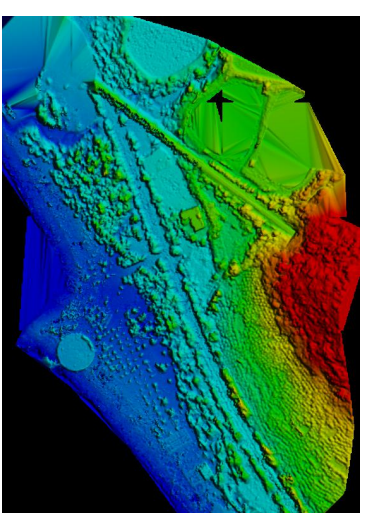

(a)DSM (colored by elevation) Figure 6. DSM and rectified image produced by mode 4 . 


\section{CONCLUSIONS}

For the tethered balloon platform, the nearly fixed positions of the camera stations are very disadvantageous to the image processing. Indeed, the image processing of high precision aerial triangulation is related to the baselines of the camera station.

The mapping system and data processing method introduced in this paper are improved in two aspects in order to adapt to the tethered balloon platform. By adding auxiliary cameras and taking images of different heights for data processing, the problem of small base-to-height ratio could be solved. Point cloud and orthophoto can be made by the mapping system for the tethered balloon.

\section{ACKNOWLEDGEMENTS}

This work was partly supported by the Strategic Priority Research Program of the Chinese Academy of Sciences (Grant No. XDA13030402), and partly supported by the Bureau of International Co-operation Chinese Academy of Sciences (Grant No. 181811KYSB20160040).

\section{REFERENCES}

Altan, M.O., Celikoyan, T.M., Kemper, G., Toz, G.K., 2008. Balloon photogrammetry for cultural heritage. In: International Archives of the Photogrammetry, Remote Sensing and Spatial Information Sciences, Istanbul, Turkey, (35), Part B5.

Bitelli, G, Girelli V A, Tini M A, Vittuari L, 2004 Low height aerial imagery and digital photogrammetrical processing for archaeological mapping. Remote Sensing and Spatial Information Sciences, 35(B5), pp. 498-503.

Gui, D.Z., Lin, Z.J., Liu, Z.Q., Peng, X.D., Li, G.S., Xie, F.F., 2009. Mosaic model of compact and wide-angle composite digital camera system for UAV. Infrared and Laser Engineering, 38(5), pp. 905-914.

Mozas-Calvache, A.T., Perez-Garcia, J.L., Cardenal-Escarcena, F.J., Mata-Castro, E., Delgado-Garcia, J., 2012. Method for photogrammetric surveying of archaeological sites with light aerial platforms. Journal of Archaeological Science, 39, 521530 .

Peng, G.L., Wan, Z.Q., 2019. The present situation and prospect of aerostat applied to remote sensing and remote survey in China. Journal of Geo-information Science, 21 (4), 504-511.

Peng, X.D., Lin, Z.J., 2009. Unmanned airship low altitude system for aerial photogrammetry. Science of Surveying and Mapping, 34(4), 11-14

Ren, P., Meng, Q.L., Zhang, Y.F., Zhao, L.H., Yuan, X., Feng, X.H., 2015. An unmanned airship thermal infrared remote sensing system for low-altitude and high spatial resolution monitoring of urban thermal environments: integration and an experiment. Remote Sensing, 7, 14259-14275.

Zhang, A.W., Hu, S.X., Meng, X.G., Yang, L.B., Li, H.L., 2015. Toward high altitude airship ground-based bore sight calibration of hyperspectral push broom imaging sensors. Remote Sensing, 7, 17297-17311.

Zhang, Y.J., 2009. Geometric Processing of low altitude remote sensing images captured by unmanned airship.Geometrics and Information Science of Wuhan University, 34(3), 284-287. 